\title{
Core and Edge Confinement Studies with Different Heating Methods in JET
}

\author{
JET Team ( prepared by F.G. Rimini $^{1}$ and G. Saibene ${ }^{2}$ ) \\ JET, Joint Undertaking, Abingdon, Oxfordshire, United Kingdom
}

\author{
present address : \\ ${ }^{1}$ Ass. EURATOM-CEA sur la Fusion - CEN Cadarache, St. Paul-lez-Durance, France \\ ${ }^{2}$ EFDA Close Support Unit, Garching, Germany
}

E-mail contact of F.G. Rimini: rimini@drfc.cad.cea.fr

\begin{abstract}
A detailed comparison of core and edge confinement with different heating methods, NBI and ICRH, has been carried out in the ELMy H-mode regime in JET with the Gas Box divertor. Transport in the core and characteristics of the edge pedestal have been assessed in discharges at 2.0 MA / 2.6 T at total input power level of 11-12 MW. The thermal core confinement has been found to be higher by about 10\% in ICRH dominated discharge. Although this difference is well within the uncertainties of the thermal confinement estimation, it has been consistently found in similar experiments in the past and it may be related to the more peaked power deposition provided by ICRF heating. Local transport analysis carried out with the TRANSP code indicates that, independently of the NBI vs. ICRH mix, ion conduction losses are the dominant energy loss channel. Unlike previous experiments, the gas flow and density of NBI and ICRH discharges have been closely matched. In these conditions it has been found that both types of heating yield similar values of edge density and temperature and produce similar ELMs. The fact that the edge fast ion concentration can be varied from roughly $0.4 \%$ up to $4 \%$ without producing significant changes in the edge pedestal parameters is an indication that fast ions do not always play a dominant role in the edge stabilization between ELMs, especially in discharges with strong gas fuelling.
\end{abstract}

\section{Introduction}

The tokamak ELMy H-mode regime is the most developed and best documented amongst the scenarios foreseen for the operation of a future tokamak reactor experiment [1]. Nevertheless, the extrapolation from the basic scenario, as observed in the present generation of tokamaks, to a reactor size machine with dominant electron heating via alpha particle slowing down is not straightforward. One of the important issues in this respect is the characterization of core confinement and edge transport barrier with different types of additional heating. In the majority of high power experiments in present divertor configurations the additional power is mainly provided by Neutral Beam Injection (NBI) at energies $\approx 100 \mathrm{keV}$, which supplies at the same time predominantly ion heating, toroidal momentum and fuelling. On the other hand, Ion Cyclotron Resonance Heating (ICRH), via acceleration of ions by absorption at fundamental or higher harmonic resonance, can deliver either ion or electron heating with no fuelling and minimal or zero toroidal momentum input. An alpha particle heating dominated plasma will have propertiesmore similar to ICRF-heated plasmas in present tokamak devices. The alpha particle slowing-down will yield electron heating with a large fast ion population. In addition, in a driven fusion device, a high-energy NBI system may give a combination of ion and electron heating with reduced fuelling and momentum input compared to present experiments, while an ICRF heating system can be tuned to give dominant electron or ion heating [2].

The JET tokamak is eminently placed to contribute to studies of reactor-relevant ELMy Hmode plasmas with varying mixture of NBI and ICRF heating. Firstly it has multi-megawatt

\footnotetext{
${ }^{\#}$ see appendix to IAEA-CN-77/OV1/2, The Jet Team (Presented by. C. Gormezano)
} 
NBI and ICRF systems and a closed divertor. Secondly in scenarios with dominant ICRF heating, tuned for example to hydrogen minority resonance $(\mathrm{H}) \mathrm{D}$, a high energy tail develops in the ion minority distribution function, which extends into the megaelectronvolt energy range. These fast ions, thanks to JET's large size and the relatively high plasma current are well confined, thereby providing a simulation of alpha particle heating in a reactor-type plasma.

\section{Experiment Overview}

In order to assess differences in core and edge confinement, a series of ELMy H-mode discharges was carried out at $2 \mathrm{MA} / 2.6-2.7 \mathrm{~T}$ in single -null, moderate triangularity $\delta \approx 0.25$, configuration in the GasBox divertor at constant input power, $\sim 11 \mathrm{MW}$, and varying proportion of NBI and ICRH power (FIG. 1) [3]. The toroidal field value was chosen so as to minimise the $\mathrm{L}-\mathrm{H}$ power threshold with respect to available ICRH power. For the discharges analysed in this paper the $\mathrm{L}-\mathrm{H}$ power threshold was estimated to be in the range of 5-6 MW. The plasma current was limited to $2 \mathrm{MA}$ in order to avoid large sawteeth and the appearance of confinement degrading MHD activity at the crash of such sawteeth, as is sometimes observed at high ICRH power.

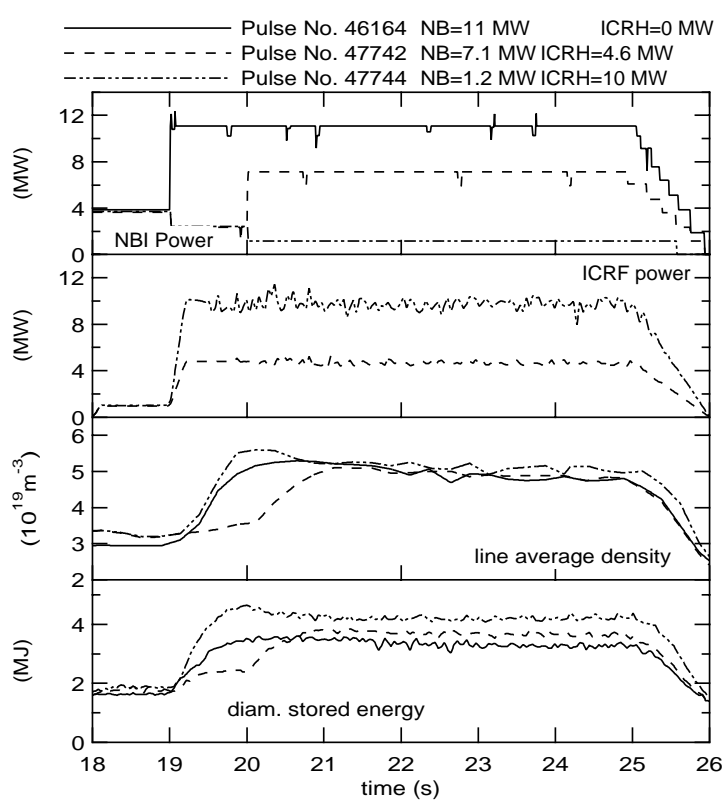

Figure 1: time evolution of plasma parameters for the extreme and mid points of the scan

The scan extended from pure NBI heating to a mixture of $10 \mathrm{MW}$ of ICRH power plus $\sim 1$ MW of NBI power, which is the minimum necessary for ion temperature measurements via Charge Exchange Recombination Spectroscopy (CXRS). All ICRH antennas were tuned to the same frequency of $\sim 42 \mathrm{MHz}$ corresponding to the fundamental hydrogen minority resonance at normalised radius $\rho \sim 0.2$ on the high field side.

One of the main differences with respect to similar previous experiments in JET was in the control of the gas fuelling, and in particular in the use of external gas fuelling throughout the ELMy H-mode phase. The constant gas fuelling was provided by a mixture of pure $\mathrm{D}_{2}$ and $\mathrm{D}_{2}+10 \% \mathrm{H}_{2}$ at the total rate of $\sim 1 \times 10^{22} \mathrm{el} . / \mathrm{s}$. This is to be compared with a fuelling from the NBI of $\sim 1 \times 10^{20} \mathrm{el} . / \mathrm{s}$ in the mainly ICRH case and up to $\sim 1 \times 10^{21} \mathrm{el} . / \mathrm{s}$ in the NBI only case. The constant mixed gas fuelling has a dual purpose: it allows a good match of core and edge density to be achieved throughout the scan (FIGURES 1 and 2) and it maintains a level of $\sim 4 \%$ of hydrogen concentration, as measured in the edge plasma region. With high levels of ICRH power, control of the hydrogen concentration in the range of $5 \%$ is desirable in order to minimise fast ion acceleration, and thereby the radial displacement of fast ions, limit fast ion orbit losses and keep a centrally peaked power deposition profile. 

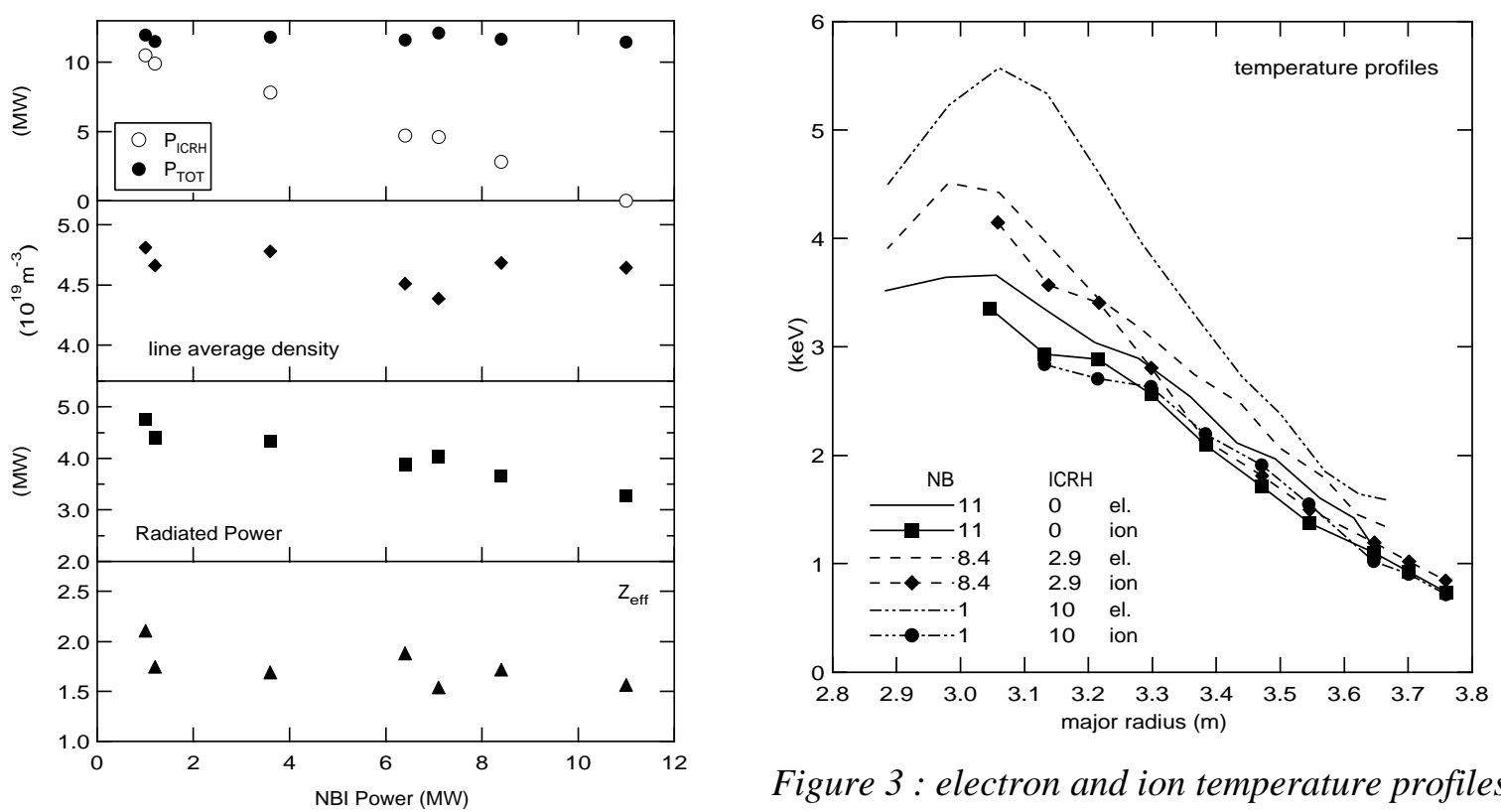

Figure 3 : electron and ion temperature profiles

Figure 2 : overview of input and ICRF power, for selected discharges in the scan at $t \approx 23 \mathrm{~s}$ density, radiation and Zeff at $t \approx 23$ s

\section{Core Confinement}

Throughout the scan the density was kept constant in value and profile shape at a level of $4.6-4.8 \times 10^{19} \mathrm{~m}^{-3}$ of line average density (FIG. 2), corresponding to $\sim 70 \%$ of the Greenwald density limit $\mathrm{n}_{\mathrm{GDL}}$. With increasing fraction of ICRF power the discharges exhibit a modest increase in the radiation level and impurity content : the total radiated power goes from $25 \%$ to $\sim 35 \%$ of the input power and $Z_{\text {eff }}$, from 1.5 to 2 (FIG. 2).

The higher ICRF power fraction results in an increase of electron temperature in the core region (FIGURES 3 and 4), accompanied by a stronger sawtooth activity. Interestingly, the core ion temperature increases as well, up to roughly the point of equal NBI and ICRF heating (FIGURES 3 and 4), decreasing eventually in the highest ICRF power case. Profiles of electron temperature and, to a lesser extent, ion temperature tend to become more peaked with increasing ICRF power.

All discharges are in H-mode regime and maintain steady confinement for approximately 10 times the total energy confinement time. The duration of the H-mode phase is limited only by the available length of the heating pulse. The global confinement reflects the increased core temperature with ICRF power: thermal stored energy and normalised confinement factor H97 increase by about $10 \%$ (FIG. 4). It has to be pointed out that differences of such magnitude are within the error bars of the estimate of the thermal energy component from the measured diamagnetic energy and the fast ion energy component computed by the CHEAP code [4] for the NBI ions and by the PION code [5] for the fast ions in the combined ICRH+NBI cases. Nevertheless it also has to be stressed that the observed increased thermal confinement is in line with previous JET experiments and numerical modelling [6] showing that the confinement of ICRF heated plasmas is improved by the more central power deposition compared with the NBI power deposition in these relatively high density conditions. 


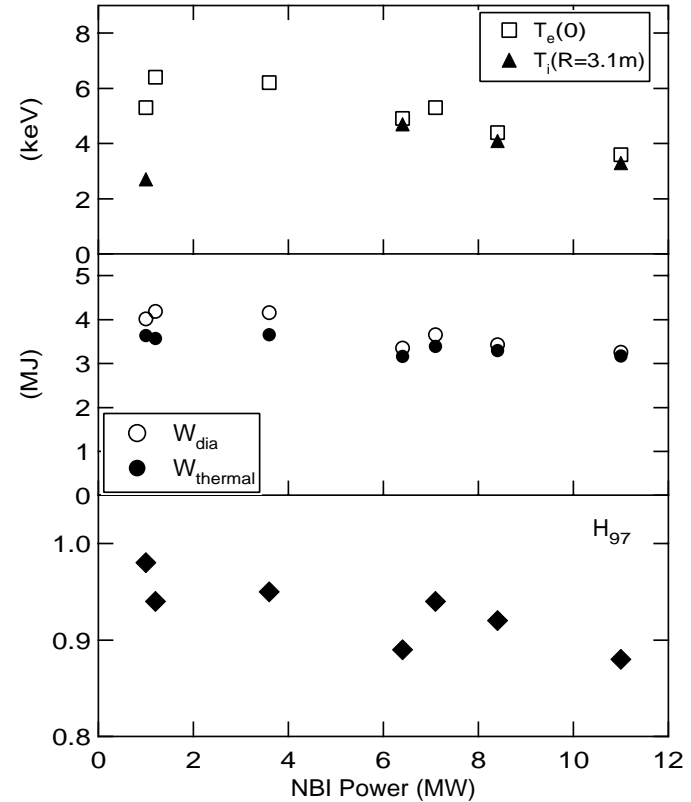

Figure 4: core confinement overview - data taken at $t \approx 23 \mathrm{~s}$

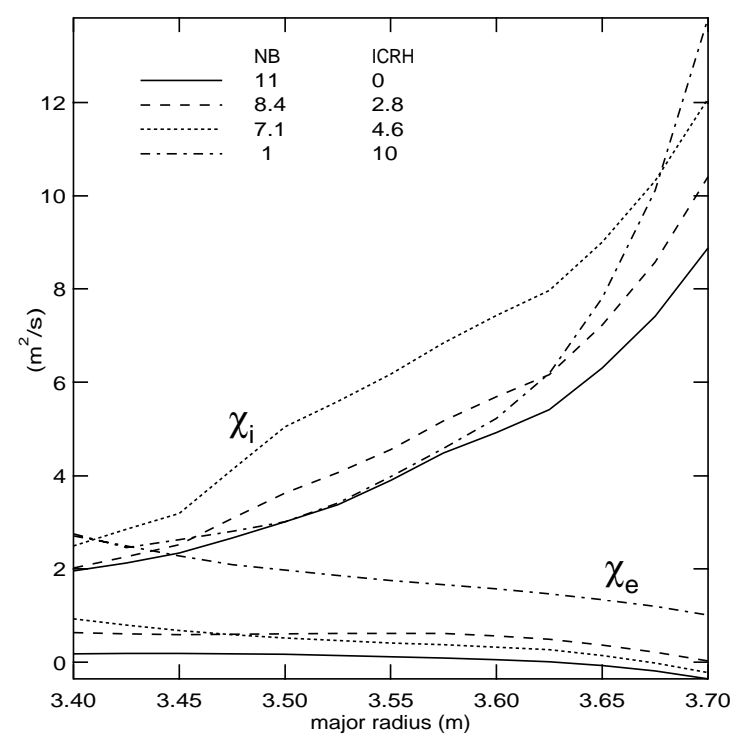

Figure 5 : electron and ion heat diffusivities at $t \approx 23 s$ (TRANSP code)

Local transport analysis carried out with the TRANSP code [7] shows that the ion thermal diffusivity profiles are independent of the proportion of ICRF and NBI power (FIG. 5). Ion conduction is the dominant energy loss channel in all cases; electron transport is enhanced at high ICRF power levels, but it remains smaller than ion transport and it does not significantly affect the global energy balance. In the NBI only and up to 50:50 NBI+ICRH the absorbed power is split almost equally between ions and electrons, with increasing peaking of the profiles with ICRF power. In the ICRH dominated cases the power absorbed by bulk electrons is more than twice the power transferred to bulk ions. The observed increase of core ion temperature is likely to be correlated with the increasing central power deposition and the relatively large fraction of power transferred to bulk ions with moderate ICRH power.

\section{Edge pedestal characteristics}

In previous JET ELMy H-mode experiments large differences were found in the edge barrier characteristics and in the ELM behaviour between NBI and ICRF heating [6,8]. In general it was found that, compared with NBI at similar power levels, the edge pedestal pressure was lower for on-axis ICRF heating, leading to smaller and more frequent ELMs. Typically, type III ELMs were observed with ICRH instead of low frequency, distinct type I ELMs. Since NBI heating produces a larger population of fast ions near the edge pedestal, these results were used in support of the view that type I ELMs occur at the ideal ballooning limit and the barrier width is proportional to the poloidal Larmor radius, which in turn is controlled by the ion population with the highest energy in the barrier region [9].

With respect to those earlier experiments, the discharges analysed in this paper have a few significant differences. Firstly, the power level is higher, in the region of total input power $\approx$ $12 \mathrm{MWand} \approx 9 \mathrm{MW}$ of power crossing the separatrix PSEP instead of $\approx 5-6 \mathrm{MW}$. Here $\mathrm{P}_{\mathrm{SEP}}$ is defined as the difference between the absorbed power and the bulk plasma radiation. This takes the discharges firmly above the L-H transition threshold and towards the type I ELMs and higher confinement domain [10]. Secondly, the use of gas fuelling ensures that the density 
value and profiles are kept the same independently of the heating mix, minimises the discrepancies due to predominant beam fuelling and provides similar edge conditions with respect to neutral density.

In this new set of experiments the ELM frequency and magnitude are similar for all discharges (FIG. 6). These results are in qualitative agreement with those obtained in an ICRH/NBI comparison in ELMy H-modes in ASDEX-UPGRADE [11].

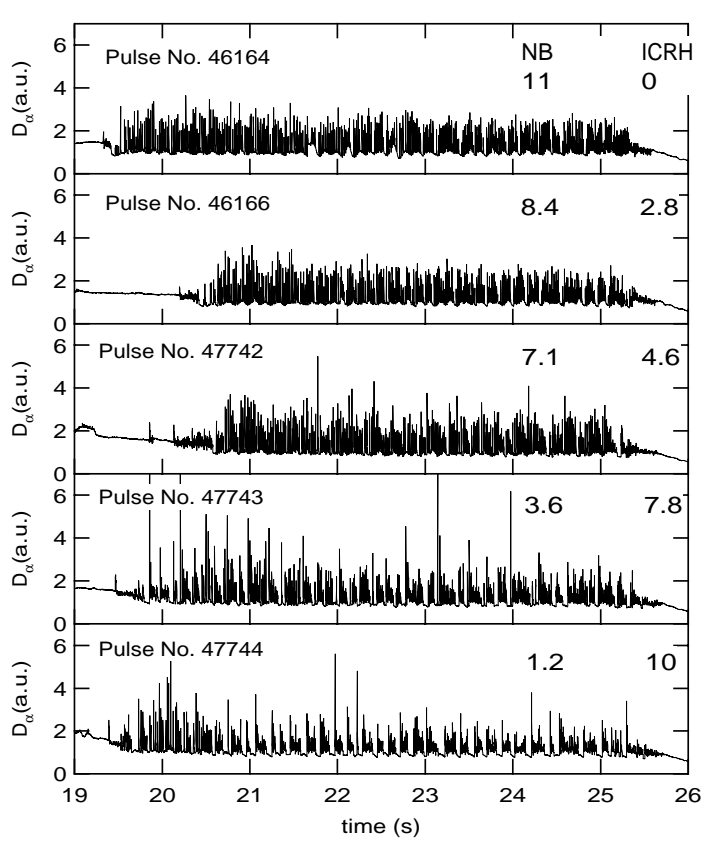

Figure 6 : Divertor Balmer- $\alpha$ overview

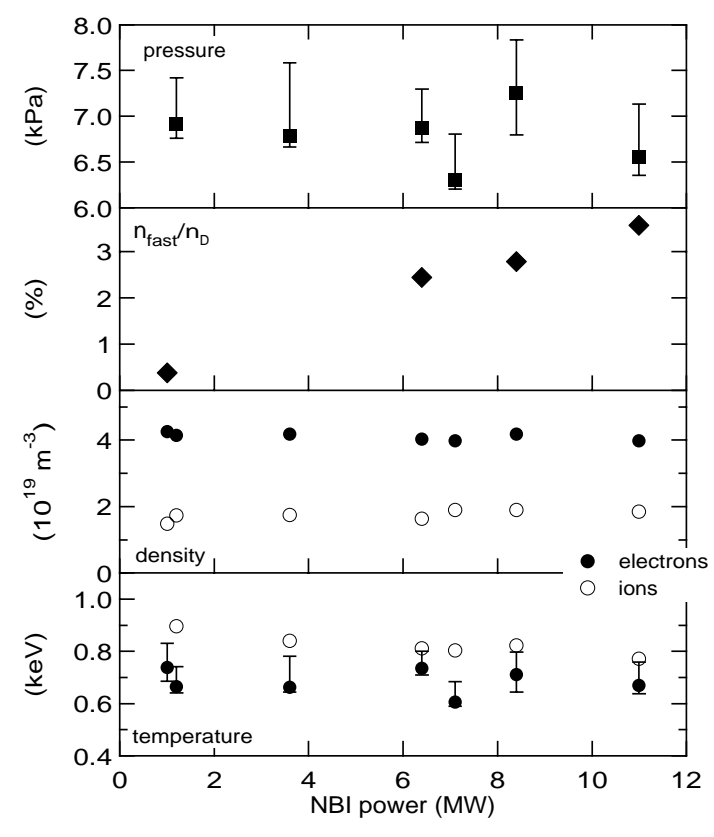

Figure 7 : overview of edge pedestal parameters.

An overview of the edge pedestal parameters is given in FIG. 7. The values of electron temperature and total pressure, $p_{\text {ped, }}$ are taken just before ELMs and the error bars indicate the ELM to ELM variation. The large variation in NBI power results in a variation of the fast ion concentration $\mathrm{n}_{\text {fast }} / \mathrm{n}_{\mathrm{D}}$ of an order of magnitude from $\sim 0.4 \%$ to $\sim 4 \%$, crossing the possible threshold of $\sim 1 \%$ for the number of fast particles necessary to influence the properties of the edge transport barrier [9]. The fast ion concentration values have to be taken with some caution since edge Charge Exchange losses of fast ions are not properly modelled in the code used, resulting in a possible overestimate of fast ion density in discharges with gas fuelling. Modelling with the PION code, including the finite orbit effects, shows that the effect of core ICRF heating on the edge fast ion population is negligible. The ICRF driven fast ion population is peaked close to the cyclotron resonance $\rho \sim 0.3$ and the fast ion component at the plasma edge is entirely determined by the NB injection. For these discharges the estimated average fast ion energy $\left\langle\mathrm{E}_{\text {fast }}\right\rangle$ in the pedestal region is in the range of $30-40 \mathrm{keV}$.

Despite the variation in edge fast ion density and energy the pedestal parameters are constant throughout the scan. The edge electron (FIG. 8) and ion temperature profiles are also very similar for the different cases. The width of the electron temperature pedestal, estimated by linear extrapolation, is in the range of $4-5 \mathrm{~cm}$ for the discharges shown. 


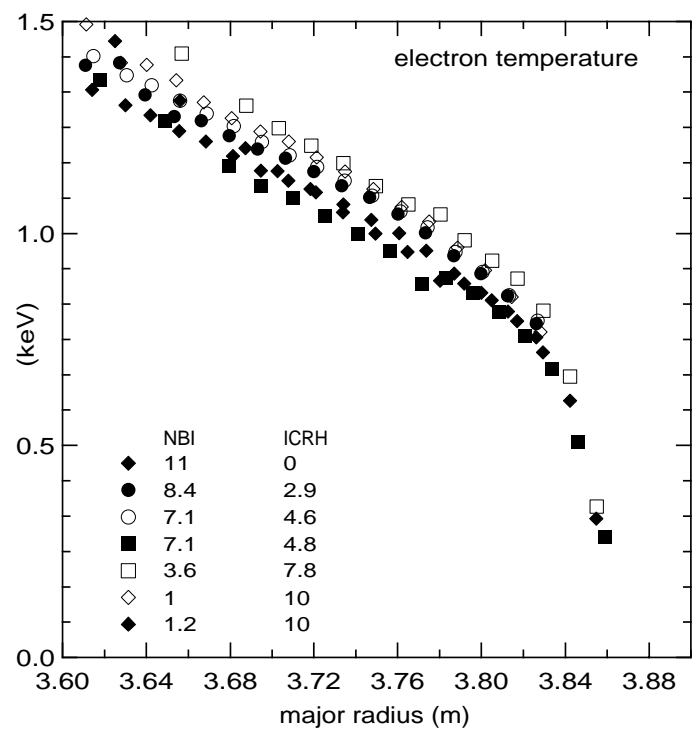

Figure 8 : edge electron temperature profiles before an ELM at $t \approx 23 s$.

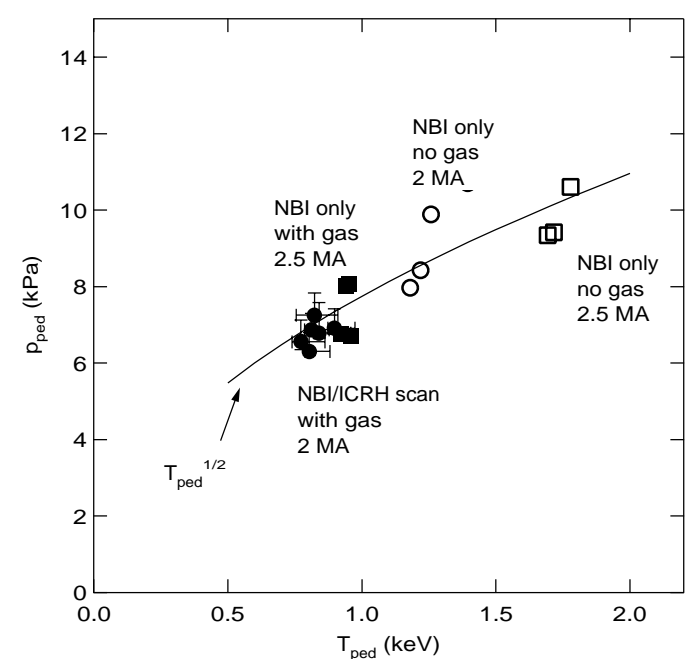

Figure 9 : pressure vs temperature at the top of a pedestal before an ELM. Data from the NBI/ICRH mixture scan are compared with NBI only data with and without gas fuelling (see text for details).

The results of the above experiments, indicated as "NB/ICRH scan with gas", are summarised in FIG. 9 in a plot of pressure vs. temperature at the top of the pedestal. The data are taken just before an ELM. For comparison data are also shown for a reference $11 \mathrm{MW}$ NBI only case with no gas fuelling and for a gas scan with NBI only at the same power level but at the higher current of $2.5 \mathrm{MA}$. The line is a curve of constant $\sqrt{\mathrm{T}_{\text {ped. }} \text {. The pedestal }}$ parameters in the present experiment are consistent with the values of the pure NB heated cases and with a scaling of pedestal pressure with $\sqrt{ } T_{\text {ped. }}$. This would imply a degradation of the edge confinement with decreasing $\sqrt{ } \mathrm{T}_{\text {ped }}[10,12]$.

Under the assumption that the maximum achievable edge pressure gradient $\nabla p_{\text {crit }}$ is determined by a unique MHD instability during the H-mode phase and in the approximation $\nabla \mathrm{p}_{\text {crit }} \sim \mathrm{p}_{\text {ped }} / \Delta_{\text {bar }}$, the evolution of the pedestal parameters and the ELMs characteristics can be interpreted as a variation of the width of the transport barrier, $\Delta_{\text {bar. }}$ In this framework the data

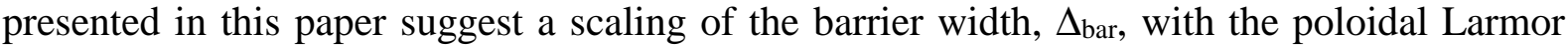
radius of the thermal component , $\rho_{\theta, \text { th }}$, rather than with the poloidal Larmor radius $\rho_{\theta, \text { fast }}$ of the ion population with the highest energy in the pedestal region.

\section{Summary and Conclusions}

In order to study the physical mechanisms controlling the characteristics of the edge transport barrier, a detailed comparison of core and edge transport in the ELMy H-mode regime has been carried out in the JET GasBox divertor at 2.0 MA / 2.6-2.7 T. The discharges in the series had varying fractions of NBI and ICRH power at total input power level of 11-12 MW and closely matched gas flow and density.

With increasing fraction of ICRF power, electron and, to a lesser extent, ion temperature increase and the temperature profiles become more peaked. The thermal core confinement is higher by about $10 \%$ in the ICRH dominated discharge. Although this difference is well within the uncertainties of the thermal confinement estimation, it has been consistently found 
in similar past experiments. The increased confinement was found to be correlated to the more peaked power deposition provided by the ICRF heating and well reproduced by numerical modelling. Throughout the scan, independently of the NBI vs. ICRH mix, ion conduction losses are the dominant energy loss channel, as indicated by the local transport analysis carried out with the TRANSP code. Although some enhancement in the electron transport is observed with increasing ICRF power fraction, electron transport remains smaller than ion transport and it does not significantly affect the global energy balance. These results on transport characteristics in ELMy H-modes with dominant electron heating, provided by slowing down of a high energy ion population, are encouraging for extrapolation to a reactor size plasma dominated by alpha particle heating.

As the proportion of NBI and ICRH power varies, no significant changes are observed in the ELM activity and in the measured values of edge pedestal parameters. The edge fast ion concentration $\mathrm{n}_{\text {fast }} / \mathrm{n}_{\mathrm{D}}$ is estimated to vary from $\sim 0.4 \%$ up to $4 \%$ at constant values of poloidal thermal Ion Larmor radius $\rho_{\theta, \text { th }}$, thus facilitating the separation of fast and thermal ion effects in the edge.

While previous JET experiments found major differences in edge characteristics between NBI and ICRF heated plasmas, the data presented in this paper suggest that, at least in regimes with external gas fuelling, the edge transport barrier parameters can be similar with NBI or on-axis ICRF minority heating. In addition, in this range of densities at $\sim 70 \%$ of $\mathrm{n}_{\mathrm{GDL}}$ the pedestal pressure before an ELM is found to be proportional to $\sqrt{ } T_{\text {ped. }}$. This is in agreement with results from JET experiments of gas fuelled NBI only ELMy H-modes [12] and isotope scans $[3,13]$.

A semi-empirical model, successfully used to interpret the earlier JET results, attributes the suppression of edge turbulence between type I ELMs to the fast ion population at the plasma edge [9]. It invokes a minimum edge fast ion concentration, $\sim 1 \%$, for the edge barrier width to be determined by the poloidal Larmor radius of the high energy ion component. This model does not seem to fit the data presented here, despite the values of the concentration $n_{\text {fast }} / n_{D}$ being in the right order of magnitude for fast ions effects to be non-negligible. Furthermore the above mentioned model does not fit other medium to high density JET ELMy H-mode results from scans of the mass of NBI injected particles and of the target plasma [3].

The investigation of the physical mechanisms controlling the edge transport barrier and the extrapolation of the edge pedestal characteristics is one of the crucial items in the studies for a next generation fusion device, such as ITER. Detailed experiments investigating the physics involved in the ELM activity have been carried out in most of today's generation of tokamaks, sometimes yielding contradictory results. The results from the heating mixture scan presented here provide a consistent set, spanning about one order of magnitude in edge fast ion concentration at constant values of thermal ion Larmor radius. They should be complemented by further experiments carried out, with a fuller complement of edge diagnostics and at higher input power levels.

\section{Acknowledgements}

The experiments discussed in this paper have been carried out in the framework of the JET, Joint Undertaking. 


\section{References}

[1] ITER Physics Expert Groups on Confinement and TRansport and Confinement Modelling and Database, ITER Physics Basis Editors, Nucl. Fusion 39 (1999) 2175

[2] Bergeaud V. et al., Nucl. Fusion 40 (2000) 35

[3] G. Saibene et al., Plasma Phys. Control. FusionVol. 23D, page 97, 1999

[4] Von Hellermann M.G. et al., in Diagnostics for Experimental Thermonuclear Fusion Reactors (Stott, P.E., Gorini, G., Sindoni, E., Eds), Plenum Press, New York and London (1996) $281 \mathrm{ff}$

[5] Eriksson, L-G. et al., Nucl. Fusion 33 (1993) 1037

[6] J. Lingertat et al., J. Nucl. Mater. 266-269 (1999) 124-130

[7] B. Balet et al., Nucl. Fusion 32 (1992) 1345

[8] V P Bhatnagar et al. Nucl. Fusion 39, p353 (1999)

[9] V.V. Parail, H.Y. Guo, J. Lingertat, Nuclear Fusion 39, p369 (1999)

[10] L.D. Horton et al. Plasma Phys. Control. Fusion 41 (1999) B329

[11] J-M. Noterdaeme et al., Proc. of 13th Topical Conf. on RF Power in Plasmas, Annapolis (USA) 1999

[12] G. Saibene et al. , Nucl. Fusion 39 (1999) 1133

[13] JET Team (presented by G. Cordey), Nucl. Fusion Yokohama Special Issue II, 39 (1999) 1763 